\title{
Hydrogen dissociation of naphthalene cations: a theoretical study
}

\author{
F. Jolibois ${ }^{1}$, A. Klotz ${ }^{2,1}$, F. X. Gadéa ${ }^{3}$, and C. Joblin ${ }^{2}$ \\ ${ }^{1}$ Laboratoire de Physique Quantique, Institut de Recherche sur les Systèmes Atomiques et Moléculaires Complexes, Université Paul Sabatier, \\ 118 route de Narbonne, 31400 Toulouse, France \\ 2 Centre d'Étude Spatiale des Rayonnements, Observatoire Midi Pyrénées, CNRS-UPS, 9 Av. du Colonel Roche, BP 4346, \\ 31028 Toulouse Cedex 4, France \\ e-mail: klotz@cesr.fr \\ 3 Groupe NanoScience, CEMES, UPR 8011, 29 rue Jeanne Marvig, BP 4347, 31055 Toulouse Cedex 4, France
}

Received 24 May 2005 / Accepted 15 June 2005

\section{ABSTRACT}

We present the computational results of removing mono and di-hydrogen atoms from the naphthalene cation. Calculations are performed at the B3LYP level with an extended basis. Two channels are studied, $-\mathrm{H}$ and $-\mathrm{H}_{2}$, and these processes are discussed within the frame of photodissociation of PAHs in the interstellar medium.

Key words. astrochemistry - molecular data - ISM: molecules

\section{Introduction}

Polycyclic Aromatic Hydrocarbons (PAHs) have been proposed as an important component of interstellar matter (Léger \& Puget 1984; Allamandola et al. 1985). They are revealed by the well-known infrared aromatic emission features in the midinfrared that are particularly strong in UV-irradiated regions. For isolated PAH molecules, infrared emission is the main relaxation process (Léger et al. 1989a). However, fragmentation may also occur when the internal energy is large enough. Several studies in the laboratory have been devoted to address this topic. Dissociative ionization experiments were performed by Leach and coworkers (Jochims et al. 1992), where highenergy VUV photons ensure both ionization and dissociation. Other data were obtained with ion trap set-ups using either photo-excitation by a laser pulse (Ho et al. 1995) or multiple visible (or near-UV) photon absorption (Boissel et al. 1997). In the latter case, an equilibrated internal energy distribution is reached as a result of heating by visible photons and cooling by infrared emission. Although the absorbed photons are at a lower energy than VUV, the approach is expected to be realistic for studying fragmentation channels at a given internal energy, since for PAHs the absorbed energy is very rapidly redistributed amongst the various degrees of freedom. Therefore only the amount of internal energy is what matters, not the way it is built.

The Boissel et al. (1997) experiments allow one to probe the lowest dissociation energy channels at rates that are in competition with infrared emission $\left(k \sim 10-10^{2} \mathrm{~s}^{-1}\right)$, whereas dissociative ionization experiments involve higher rates $\left(k \sim 10^{4} \mathrm{~s}^{-1}\right)$, and therefore can involve UV photons with energies higher than those found in HI regions, $h v<13.6 \mathrm{eV}$.

Recently, detailed experiments have been performed with the PIRENEA ion trap set-up (Joblin et al. 2002) to study the photodissociation of the coronene cation $\mathrm{C}_{24} \mathrm{H}_{12}^{+}$. These experiments show that the fragmentation process is dominated by $\mathrm{H}$ loss rather than $\mathrm{H}_{2}$ up to the formation of the bare cluster $\mathrm{C}_{24}^{+}$ (Joblin et al. 2005a). Competition between the $\mathrm{H}$ and $\mathrm{H}_{2}$ channels is of particular interest because several authors have put forward observational evidence that PAHs can play an important role in the formation of $\mathrm{H}_{2}$ in photodissociation regions (Joblin et al. 2000; Habart et al. 2004). The catalytic formation of $\mathrm{H}_{2}$ has also been theoretically predicted from the collision of $\mathrm{PAH}^{+}$(Bauschlicher 1998; Hirama et al. 2004) or $\mathrm{PAH}^{-}$ (Bauschlicher \& Bakes 2001) with atomic hydrogen. The aim of the present work is to study the $-\mathrm{H} /-\mathrm{H}_{2}$ competition formation by photodissociation of $\mathrm{PAH}^{+}$from a theoretical point of view. Since theoretical calculations are quite time-consuming for large systems, we have started here with the case of the smallest $\mathrm{PAH}$, the naphthalene cation $\mathrm{C}_{10} \mathrm{H}_{8}^{+}$.

\section{Theoretical model}

The photophysics of isolated interstellar PAH ions has been described by several authors (Léger et al. 1989a,b; Allamandola et al. 1989). A theoretical description of the dehydrogenation 


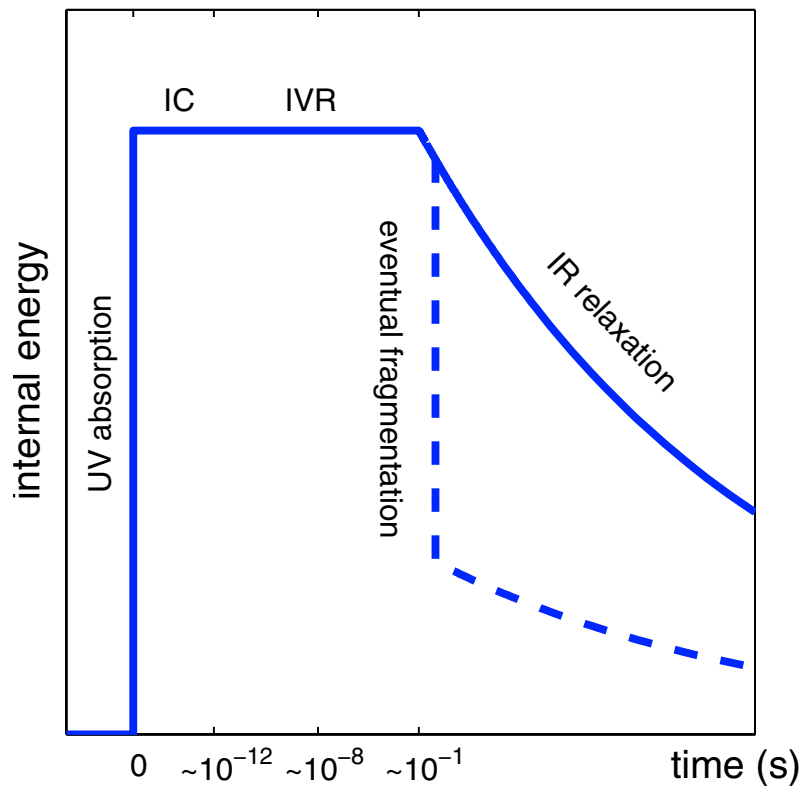

Fig. 1. Schematic view of internal energy balance in a large PAH molecule. IC is for internal conversion between electronic states. IVR is for internal vibration redistribution amongst all vibrational modes.

of PAHs in astrophysical conditions requires at least five steps: 1) electronic excitation by absorption of visible-UV photons $(\mathrm{h} v<13.6 \mathrm{eV}) ; 2$ ) evolution of the excited electronic state; 3 ) internal convertion (IC), the absorbed energy being converted from the electronic degrees of freedom to the nuclear ones, while the system recovers, at constant energy, its electronic ground state; 4) intramolecular vibrational redistribution (IVR) of the energy among all vibrational states; either 5a) if the internal energy is lower than the dissociation threshold, energy relaxation is done by infrared emission only; or else 5b) eventual fragmentation of the PAH by evaporation of small fragments $\left(\mathrm{H}, \mathrm{H}_{2}, \mathrm{C}_{2} \mathrm{H}_{2}\right.$, etc. $)$ can occur. These steps are illustrated in Fig. 1. In the first step, the molecule absorbs a given energy, while in the four other steps this energy is redistributed and relaxed. As a consequence of fast internal conversion, no fragmentation is expected when PAHs are in the electonic excited state (not the case for small molecules). For these reasons, experiments that consist of vibrational excitation of the fundamental electronic state of PAHs are relevant to mimic the excitation process expected in the interstellar medium. A complete simulation of each of the five steps described above is beyond the scope of this paper but by taking advantage of the internal conversion, we limit our study to the ground electronic state. The first three steps are then modeled by a kick in internal energy. Gradually, by increasing its energy, the molecule explores the ground-sate potential energy surface and may reach dissociative channels.

Following this scheme, we computed the ground-state Potential Energy Surface (PES) at high internal energies focusing on the limited set of internal coordinates which correspond to the dissociation channels. Our study focuses on the evaporation of $\mathrm{H}$ and $\mathrm{H}_{2}$, although other fragmentations could also

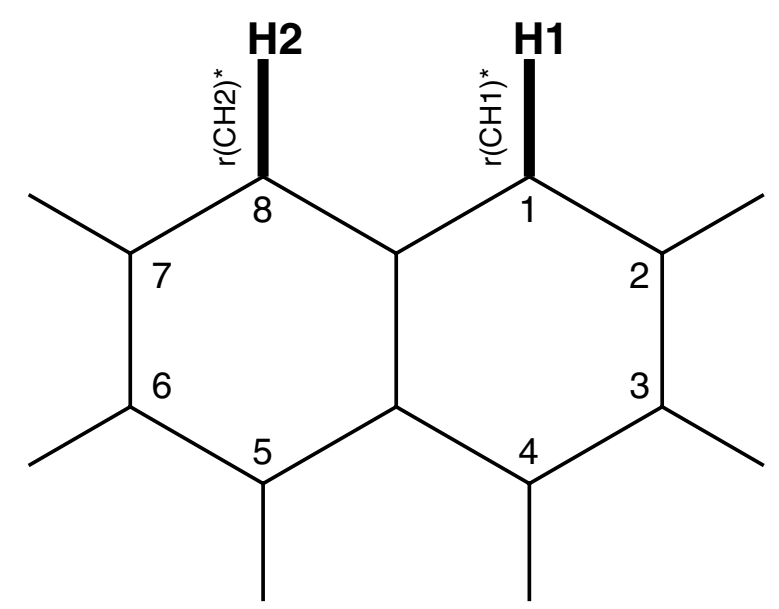

Fig. 2. Naphthalene geometry and stretching of the two apical $(1,8)$ hydrogens involved in the considered dissociative channels.

occur, in particular loss of $\mathrm{C}_{2} \mathrm{H}_{2}$ (Jochims et al. 1992; Gotkis et al. 1993; Granucci et al. 1995).

\section{The calculations}

Theoretical calculations were performed for the ground state of naphthalene cation at high internal energy near the dissociation thresholds $\left(-\mathrm{H},-\mathrm{H}_{2}\right)$. We computed adiabatic potentials to obtain information regarding dissociative paths and activation barriers.

\subsection{Methodology}

We used two reaction coordinates defined by the stretching of two adjacent apical $(1,8)$ hydrogens (hereafter $\mathrm{H} 1$ and $\mathrm{H} 2$ ) of the naphthalene cation (see Fig. 2). We chose them rather than other hydrogens because the simultaneous stretch of their bonds keeps a short distance between $\mathrm{H} 1$ and $\mathrm{H} 2$ and favours the $\mathrm{H}_{2}$ molecule formation. After the loss of $\mathrm{H} 1$ and $\mathrm{H} 2$, we supposed that one adjacent hydrogen ( 2 or 7 ) possibly performs a low energetical rearrangement ( 7 to 1 , or 2 to 8 ) to form a naphth-1-yne compound. In this paper, this rearrangement is not studied because tunneling processes and spin problems may occur and the computational level used is not adapted to treat such rearrangement.

Quantum chemistry calculations were performed for each pair of stretches $r(\mathrm{CH} 1)$ and $r(\mathrm{CH} 2)$. For a given pair, with the entire geometry of the ion is optimized, the two stretched coordinates $r(\mathrm{CH} 1), r(\mathrm{CH} 2)$ remaining frozen. As a consequence, the two bonds, $\mathrm{CH} 1$ and $\mathrm{CH} 2$, not longer remain parallel due to the relaxation caused by geometry optimization. We denoted the distances for the relaxed geometries $r(\mathrm{CH} 1)^{*}$ and $r(\mathrm{CH} 2)^{*}$, as well as the stretch length between $r(\mathrm{CH} 1)^{*}$ and its value for the equilibrium state geometry, $\Delta r(\mathrm{CH} 1)$. A similar definition was used for $\Delta r(\mathrm{CH} 2)$.

We computed all geometry-relaxed conformers that correspond to $\mathrm{CH}$ stretchings varing between 0 and $3 \AA$ with a step of $0.2 \AA$ for $\mathrm{H} 1$ and $\mathrm{H} 2$. Calculations were performed using the hybrid density functional method (DFT): Becke's three-parameter non-local exchange functional (Becke 1992) 


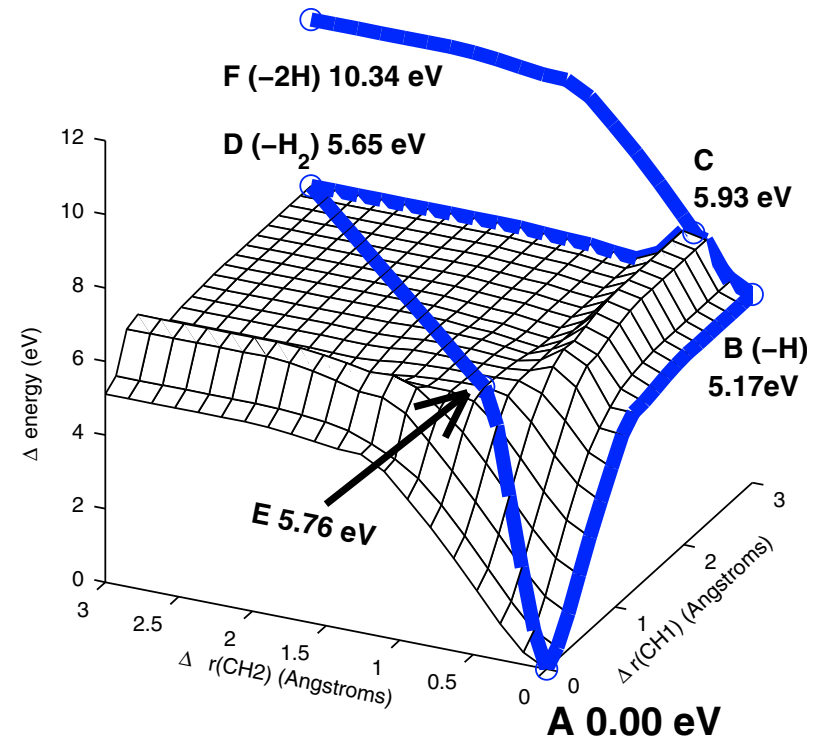

Fig. 3. Potential surface for the stretched $\mathrm{C}_{10} \mathrm{H}_{8}{ }^{+}$. $\mathrm{H} 1$ and $\mathrm{H} 2$ design the two apical adjacent hydrogens (cf. Fig. 2). Axes are stretches $\Delta r(\mathrm{CH})$ for $\mathrm{H} 1$ and $\mathrm{H} 2$. Bold solid lines are different channels discussed in the text. $\mathrm{D}$ and $\mathrm{F}$ points correspond to asymptotic potentials for $-\mathrm{H}_{2}$ and $-2 \mathrm{H}$ paths respectively.

with the non-local correlation functional of Lee, Yang, and Parr (1988, hereafter B3LYP). This method is known to give good results for energies and for vibrational analysis of polycyclic aromatic hydrocarbons (see Langhoff 1996 or Bauschlicher et al. 1999). All species are computed as doublet states except for 1-naphthyl, which was computed in its singlet and triplet states. We used the DFT implementation in the software Gaussian98 (Frisch et al. 1998) and a $6-31 G^{* *}$ basis that includes polarisation functions on both $\mathrm{C}$ and $\mathrm{H}$ atoms.

Potential surface is presented in Fig. 3. Particular conformations, noted by capital letters, are summarized in Table 1. It is important to notice that the energies of the potential maps are not corrected by the Zero Point vibrational Energy (ZPE). ZPE is only computable for minimal energy conformations, which

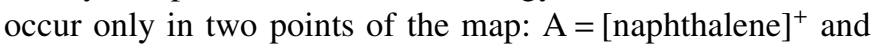
when $(\Delta(\mathrm{CH} 1)=\infty, \Delta(\mathrm{CH} 2)=0)$, corresponding to the [napth$1-\mathrm{yl}]^{+}$(designed $\mathrm{B}_{\infty}$ ). For these two species we also calculated the infrared spectrum (Fig. 4). Scott \& Radom (1996) fitted values for the scaling factor to convert theoretical vibrational frequencies into the experimental ones. Unfortunately, they study the B3LYP, 6-31G* method but not the B3LYP, 6-31G** we used. In the absence of study for B3LYP, 6$31 \mathrm{G}^{* *}$, we used the scaling factor of B3LYP, 6-31G*: 0.9614 $\left(\mathrm{rms} 34 \mathrm{~cm}^{-1}\right)$. Anyway, only very small differences are expected (less than 1\%) and there is no effect on the main results of this study.

In addition, we describe some specific channels corresponding to peculiar cases. We show the presence of metathesis of $\mathrm{H}_{2}$ on a carbon center that appears when the two hydrogens are connected to the same carbon (Fig. 5). A vibrational analysis was performed for configurations $\mathrm{A}, \mathrm{H}$, and $\mathrm{L}$ from the potentials given by the DFT computations. We present the infrared spectra of these compounds in Fig. 6 using the scaling factor 0.9614 .

\subsection{Analysis of the -H channel}

The -H channel corresponds to the stretch of only one coordinate (path $\mathrm{AB}$ in Fig. 3). The energy increases almost linearly up to $4.5 \mathrm{eV}$ until $\Delta r(\mathrm{CH})$ of about $1.2 \AA$. For larger stretches, the energy reaches an asymptotic value of $5.17 \mathrm{eV}$ at $3 \AA$. We computed the asymptotic energy $\left(\mathrm{B}_{\infty}\right)$ as that of [1-naphthyl] ${ }^{+}$ plus the isolated $\mathrm{H}$ energy. In that case, it is possible to perform the vibrational analysis of [1-naphthyl $]^{+}$and to determine the ZPE for the singlet ${ }^{1} \mathrm{~A}^{\prime}$ and triplet ${ }^{3} \mathrm{~A}^{\prime \prime}$. These energies are reported in Table 1 for the lines labelled $\left(\mathrm{B}_{\infty}\right)$. The $D_{\mathrm{o}}$ energy we found for the ${ }^{1} \mathrm{~A}^{\prime}$ state $(4.89 \mathrm{eV})$ and the ${ }^{3} \mathrm{~A}^{\prime \prime}$ state $(4.84 \mathrm{eV})$ are almost equal and are similar to that found by Bauschlicher (1998). A value of $4.48 \pm 0.10 \mathrm{eV}$ was derived from experimental data by Ho et al. (1995). The experimental and theoretical values are in reasonable agreement, considering the associated uncertainties.

Figure 4 shows the dramatic changes of the spectra when comparing the singlet and triplet states of [1-naphthyl] ${ }^{+}$. As already discussed by Pauzat et al. (1993), the spectrum of the triplet state is close to the [naphthalene] ${ }^{+}$one and the spectrum of the singlet state is close to that of neutral naphthalene. We note that the two states are so close in energy that a multiconfiguration computation should be performed to describe the ground state of the [1-naphthyl $]^{+}$precisely.

\subsection{Analysis of the $-\mathrm{H}_{2}$ concerted channel}

The $-\mathrm{H}_{2}$ direct channel corresponds to the stretch of both coordinates at the same time (path AD in Fig. 3). The energy increases to $5.76 \mathrm{eV}$ until $\Delta r(\mathrm{CH} 1)=\Delta r(\mathrm{CH} 2)=1.6 \AA$ (point E). For larger stretches, the energy decreases and reaches an asymptote at $5.65 \mathrm{eV}$ (point $\mathrm{D}$ ). We note that the potential is very flat, like a plateau, behind the saddle point $\mathrm{E}$.

\subsection{Analysis of the $-\mathrm{H}_{2}$ non-concerted channel}

This channel consists of two steps: first a large stretching for $\mathrm{H} 1$ (but no dissociation) followed by a stretching for $\mathrm{H} 2$. It is not an energetically favorable path, but it does illustrate a sequential path. After reaching conformation $\mathrm{B}$ at $\Delta r(\mathrm{CH} 1)=3.0 \AA$ and $\Delta r(\mathrm{CH} 2)=0$, only the second stretch increases (path BD in Fig. 3). An extra energy of $0.76 \mathrm{eV}$ is needed at $\Delta r(\mathrm{CH} 2) \sim 0.4 \AA$ (point $\mathrm{C}$ ). After that activation barrier, the energy falls down to the plateau of $\mathrm{H}_{2}$ formation. Realistic channels for $\mathrm{H}_{2}$ formation should lie between paths AED and ABD. The barrier to form $\mathrm{H}_{2}$ is higher by $0.59 \mathrm{eV}$ $\left(\Delta E_{E-B}\right)$ or $0.76 \mathrm{eV}\left(\Delta E_{C-B}\right)$ compared to the $\mathrm{B}$ energy level of the $-\mathrm{H}$ channel.

\subsection{Analysis of the $-2 \mathrm{H}$ sequential channel}

The $-2 \mathrm{H}$ sequential channel consists of dissociating $\mathrm{H} 1$ (represented by B) and then $\mathrm{H} 2$ (represented by F). This channel 
Table 1. Geometrical and energetical properties for particular geometries designated by capital letters. The $\Delta$ energy column gives results from the DFT B3LYP 6-31G** without Zero Point vibrational Energy (ZPE) correction. The $\triangle \mathrm{ZPE}$ column gives ZPE difference compared to the A conformation. The $D_{\mathrm{o}}$ column provides the corrected value ( $\Delta$ energy $\left.-\triangle \mathrm{ZPE}\right) . D_{\mathrm{o}}{ }^{*}$ is computed by Bauschlicher (1998).

\begin{tabular}{cccccccc}
\hline \hline Symbol & Designation & $\begin{array}{c}\Delta r(\mathrm{CH} 1) \\
\AA\end{array}$ & $\begin{array}{c}\Delta r(\mathrm{CH} 2) \\
\AA\end{array}$ & $\begin{array}{c}\Delta \text { energy } \\
\mathrm{eV}\end{array}$ & $\begin{array}{c}\Delta \mathrm{ZPE} \\
\mathrm{eV}\end{array}$ & $\begin{array}{c}D_{\mathrm{o}} \\
\mathrm{eV}\end{array}$ & $\begin{array}{c}D_{\mathrm{o}}{ }^{*} \\
\mathrm{eV}\end{array}$ \\
\hline $\mathrm{A}$ & [naphthalene] $^{+}$ & 0.0 & 0.0 & 0.00 & 0.00 & 0.00 & 0.00 \\
$\mathrm{~B}$ & $-\mathrm{H}$ & 0.0 & 3.0 & +5.17 & & & \\
$\mathrm{~B}_{\infty}$ singlet & {$[1 \text {-naphthyl] }]^{+}+\mathrm{H}$} & 0.0 & $\infty$ & +5.29 & -0.43 & +4.86 & +4.89 \\
$\mathrm{~B}_{\infty}$ triplet & [1-naphthyl] $^{+}+\mathrm{H}$ & 0.0 & $\infty$ & +5.19 & -0.35 & +4.84 & +4.84 \\
$\mathrm{C}$ & barrier & 0.4 & 3.0 & +5.93 & & & \\
$\mathrm{D}$ & $-\mathrm{H}_{2}$ & 3.0 & 3.0 & +5.65 & & & \\
$\mathrm{E}$ & saddle point & 0.8 & 0.8 & +5.76 & & & \\
$\mathrm{~F}$ & $-2 \mathrm{H}$ & 3.0 & 3.0 & +10.34 & & & \\
\hline
\end{tabular}
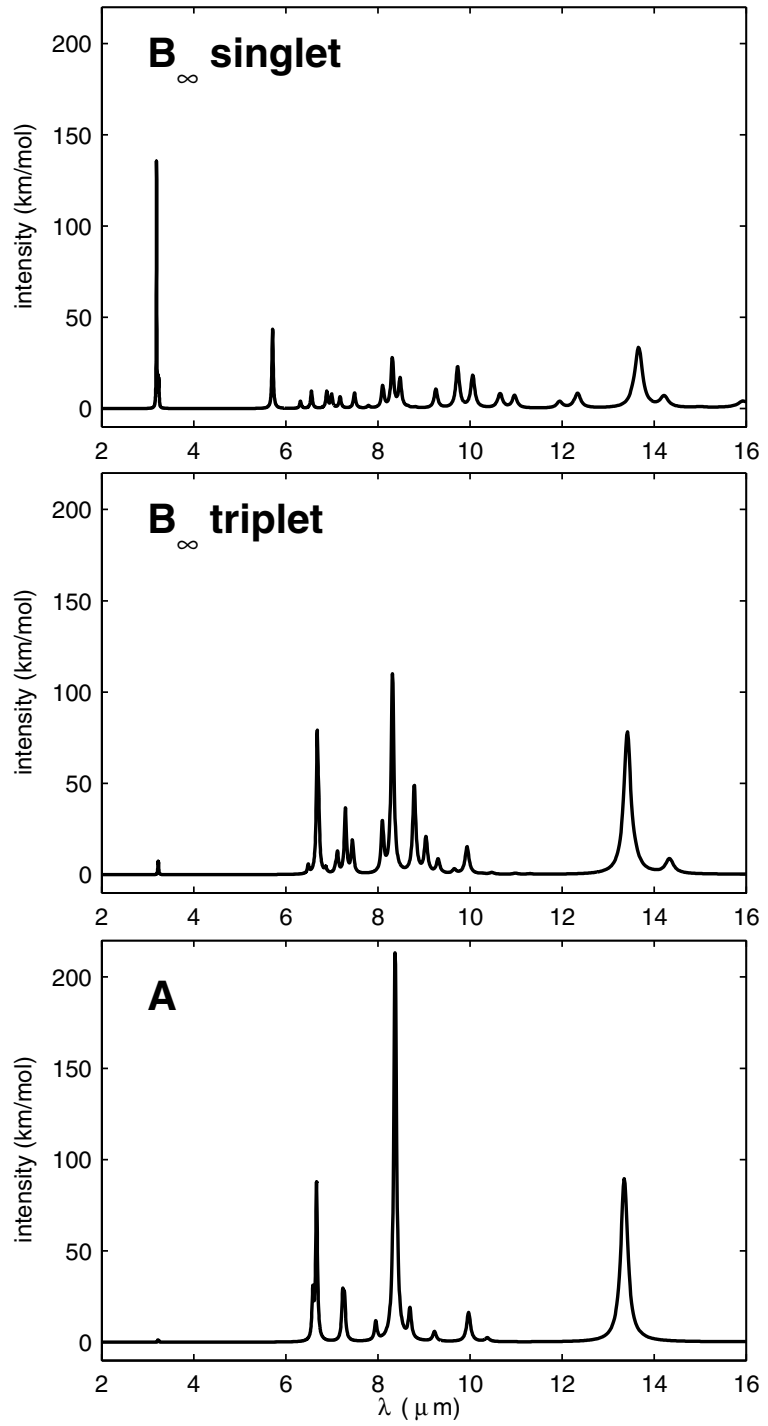

Fig. 4. Infrared spectra computed from DFT for species defined in Table 1. Frequencies were scaled by a factor of 0.9614 according to Scott \& Radom (1996). Lines were broadened by lorentzian of $10 \mathrm{~cm}^{-1}$ to look like real spectra.

is illustrated in Fig. 3 by the path ABCF. Since the energy difference $\mathrm{B}-\mathrm{F}$ is equal to the energy difference $\mathrm{A}-\mathrm{B}$, we assumed the same shape of the potential curve (path BF in Fig. 3). Important is that no activation barrier is encountered. The energy difference between points $\mathrm{F}$ and $\mathrm{D}$ $(10.34-5.65=4.79 \mathrm{eV})$ corrected by the $\mathrm{ZPE}$ of $\mathrm{H}_{2}(0.28 \mathrm{eV}$ from our computations) is characteristic of the bonding energy of the $\mathrm{H}_{2}$ molecule (4.52 eV, Huber \& Hertzberg 1979).

\subsection{Metathesis}

During this exploratory work, we found another interesting mechanism at energies comparable to $\mathrm{H}$ dissociation. From the initial conformation $\mathrm{A}$, bending the $\mathrm{CH} 1$ bond toward $\mathrm{CH} 2$, it was surprisingly possible to connect $\mathrm{H} 1$ to the same carbon atom as H2 (see Fig. 5). The transition state (labelled G) from A to that conformation is only $4.51 \mathrm{eV}$ high, significantly lower than all the specific points considered previously $(\mathrm{B}, \mathrm{C}, \mathrm{D}, \mathrm{E})$. It is therefore lower than the energy required to eject $\mathrm{H}$ or $\mathrm{H}_{2}$. Reaching point $\mathrm{G}$ is facilitated by the butterfly bending of the two carbon rings and by an in-plane bending of the two apical $(1,8) \mathrm{H}$. That mode occurs at $342 \mathrm{~cm}^{-1}$ but is not infrared active. The most stable conformation (labelled $\mathrm{H}$ ) is given for the hydrogens outside the naphthalene plane, one above and one below the plane. This carbon presents a $\mathrm{sp}^{3}$ hybridization. This conformation lies only at $2.20 \mathrm{eV}$ above the energy of $\mathrm{A}$. A transition state (labelled J) was found when the two hydrogens are in the naphthalene plane. Finally, we observed that one of the two hydrogens can jump relatively easily to another adjacent carbon (labelled L) through a transition state (labelled K) of $5.17 \mathrm{eV}$ relative to A and only $2.97 \mathrm{eV}$ relative to $\mathrm{H}$.

\section{Discussion}

\subsection{Stabilization of the biradical PAH cation}

As previously discussed in Sect. 3.1, the biradical 1, 8naphthdiyl cation ( $\mathrm{D}$ or $\mathrm{F}$ structure) should evolve to the naphth-1-yne cation by a hydrogen rearrangement. The activation barrier should be low due to the tunelling of hydrogens. After rearrangement, a triple $\mathrm{CC}$ bond replaces a double $\mathrm{CC}$ bond, which stabilizes the energy by about $1.75 \mathrm{eV}$ (McMillen \& Golden 1989). 


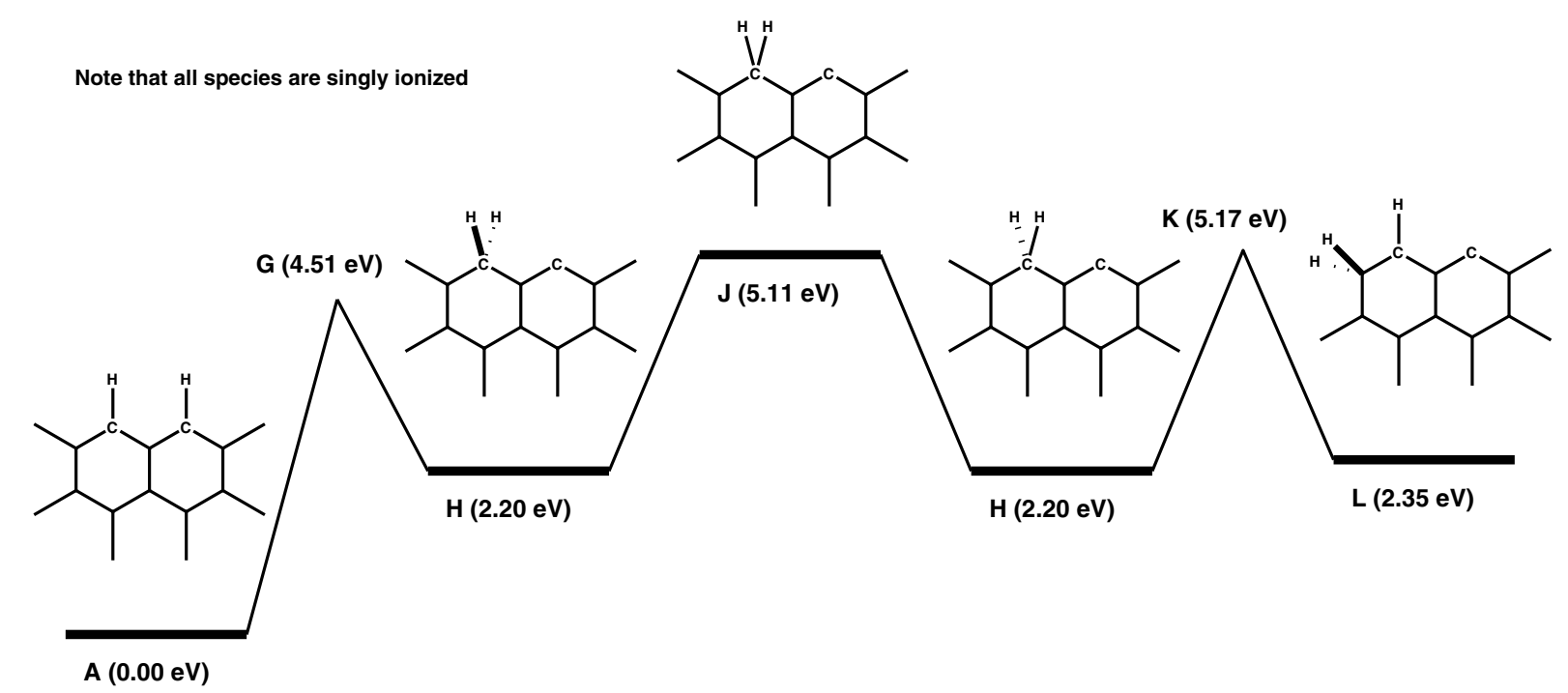

Fig. 5. Energetic pathway between the different conformers of $\mathrm{C}_{10} \mathrm{H}_{8}{ }^{+}$involved in the metathesis.
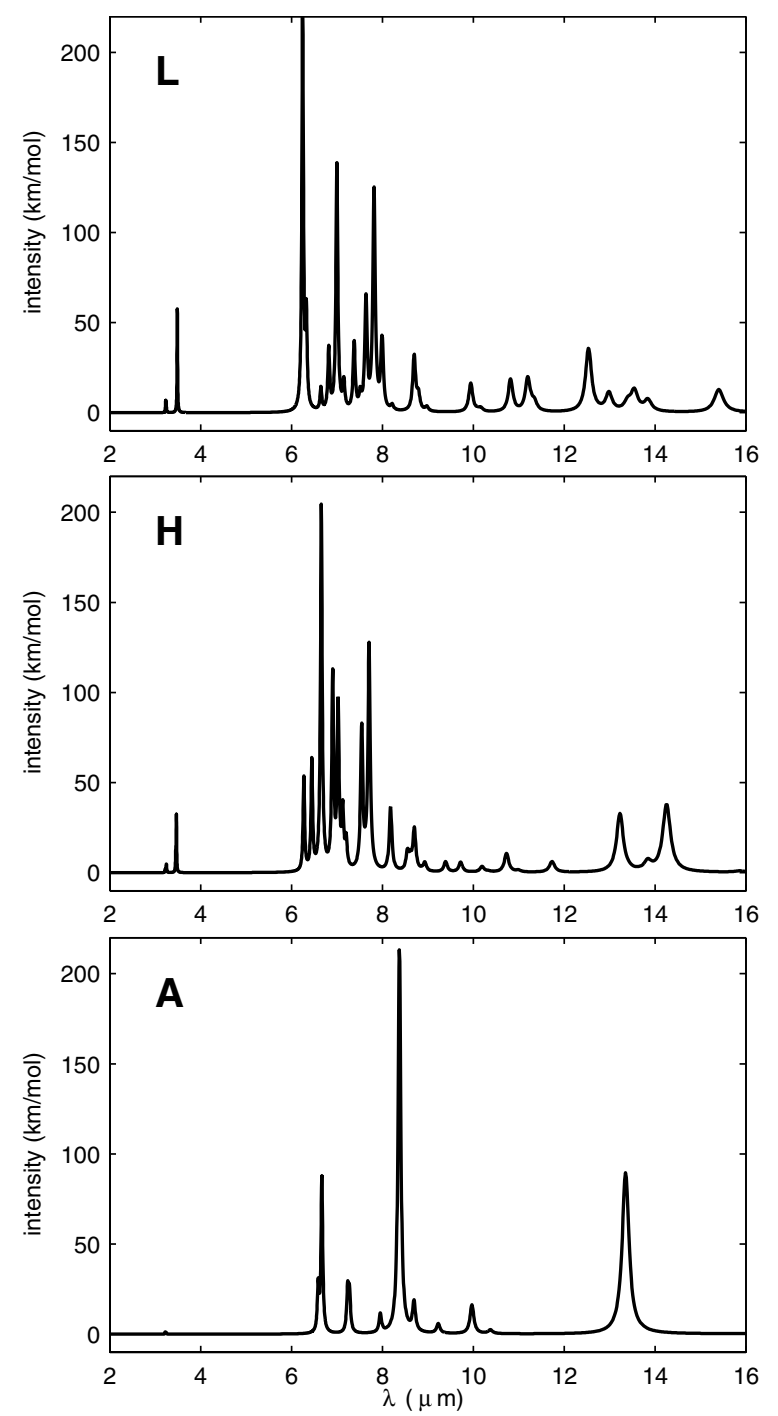

Fig. 6. Infrared spectra computed from DFT for species defined in Fig. 5. Frequencies were scaled by a factor of 0.9614 according to Scott \& Radom (1996). Lines were broadened by lorentzian of $10 \mathrm{~cm}^{-1}$ to look like real spectra.

\subsection{Competition $-\mathrm{H}$ versus $-\mathrm{H}_{2}$}

No hydrogen bond should be broken for energies lower than $4.51 \mathrm{eV}$, with $0 \mathrm{eV}$ for the ground state. Between $4.51 \mathrm{eV}$ and $5.17 \mathrm{eV}$, the methathesis channel can occur (AGH configurations), and between $5.17 \mathrm{eV}$ and $5.76 \mathrm{eV}$, the $-\mathrm{H}$ channel (AB) is allowed. For energies higher than $5.76 \mathrm{eV}$, concerted $-\mathrm{H}_{2}$ formation is also possible (AED). The repartition of the energy in specific vibrational motions should be considered, in particular for the stretching $v \mathrm{CH}$ modes involved in the breaking of the $\mathrm{CH}$ bonds. If $\mathrm{CH} 1$ and $\mathrm{CH} 2$ bond vibrations are in phase (a symmetric mode), then the vibration leads to the $-\mathrm{H}_{2}$ channel. If $\mathrm{CH} 1$ and $\mathrm{CH} 2$ bonds vibrations are in anti-phase (an asymmetric mode), then the mode may lead to the $-\mathrm{H}$ channel. In the case of the naphthalene cation, there are 4 symmetric and 4 asymmetric modes. That can be easily extended to larger PAHs. It should be noticed that asymmetric modes radiate much more internal energy than do symmetric ones but should not influence the dissociation channels because of the IVR process.

That $-\mathrm{H}$ is the lowest energy channel is consistent with the observation of mainly this channel in the PIRENEA experiment, where the increase of internal energy in the PAH is rather gradual (Joblin et al. 2005b). In other experiments, higher energy channels are accessible and the $\mathrm{H}_{2}$ channel can also be observed (Jochims et al. 1992).

\subsection{The special case of the methatesis}

Methatesis channels can occur for a very narrow range of internal energies ( $4.51 \mathrm{eV}$ to $5.17 \mathrm{eV}$ ). Figure 6 presents the infrared spectra of the A, H, L configurations. It is important to notice that the presence of only one $\mathrm{CH}_{2}$ group generates $v \mathrm{CH}$ (symmetric elongations) modes at about $3.4 \mu \mathrm{m}$ with an intensity more than ten times that of the $3.3 \mu \mathrm{m}$ band due to the ring- $\mathrm{H}$ bonds. The cisoring bend of the $\mathrm{CH}_{2}$ group generates an intense band near $7.5 \mu \mathrm{m}$.

Such PAH compounds with methathesis could be important in infrared astrophysical spectra. Since they have specific 
Table 2. Some geometries of computations for coronene cation. Values for $\Delta$ energy are listed for both naphthalene and coronene cations.

\begin{tabular}{cccc}
\hline \hline Symbol & Designation & $\begin{array}{c}\text { Naphthalene }^{+} \\
\Delta \text { energy }^{(\mathrm{eV})}\end{array}$ & $\begin{array}{c}\text { Coronene }^{+} \\
\Delta \text { energy }^{(\mathrm{eV})}\end{array}$ \\
\hline $\mathrm{A}$ & fundamental & 0.00 & 0.00 \\
$\mathrm{~B}$ & $-\mathrm{H}$ & +5.17 & +6.65 \\
$\mathrm{H}$ & vertical $\mathrm{H}_{2}$ & +2.20 & +2.40 \\
$\mathrm{~J}$ & horizontal $\mathrm{H}_{2}$ & +5.11 & +5.69 \\
\hline
\end{tabular}

signatures, it would be interesting to search for them in different environments. The band at $3.4 \mu \mathrm{m}$ has indeed been observed (Geballe et al. 1989; Joblin et al. 1996; Sloan et al. 1997) and attributed to superhydrogenated PAHs (Bernstein et al. 1996; Wagner et al. 2000) or to methyl side groups (Joblin et al. 1996). We propose that another contribution to the $3.4 \mu \mathrm{m}$ band could arise from locally superhydrogenated PAHs formed by the methathesis process emphasized above.

\subsection{Toward larger PAHs}

A few points were computed for the coronene cation $\mathrm{C}_{24} \mathrm{H}_{12}^{+}$. This computation is very long and cannot be performed for all conformations as was done for naphthalene. Results are summarized in the Table 2. Energy values are somewhat higher than those of naphthalene: however, the general trend is the same. Note that spin contamination problems occured in the calculation of the B conformer for coronene.

\section{Conclusion}

A theoretical study was performed to explore the dissociation channels $-\mathrm{H},-\mathrm{H}_{2}$ from the ground state potential energy surface of the naphthalene cation. We have found that the bond dissociation energy of the $-\mathrm{H}$ channel is lower than that of the $-\mathrm{H}_{2}$ channel by $0.57 \mathrm{eV}$. This implies that the loss of $\mathrm{H}$ is favored in the dehydrogenation process independently of the excitation mechanism. This agrees with the results obtained in the PIRENEA experiment for the dehydrogenation of the $\mathrm{C}_{24} \mathrm{H}_{p}{ }^{+}$ ( $p=[2,12])$ species by Joblin et al. (2005a,b), which suggests that the photodissociation of PAH cations cannot significantly contribute to the formation of $\mathrm{H}_{2}$ in the interstellar photodissociation regions.

An alternative catalytic mechanism proposed by Bauschlicher (1998, 1999) and Hirama et al. (2004) involves the addition of a $\mathrm{H}$ atom to the $\mathrm{PAH}^{+}$cation giving $(\mathrm{PAH}) \mathrm{H}^{+}$cation. The arrival of another $\mathrm{H}$ atom leads to the formation of $\mathrm{H}_{2}$ and to release of the initial $\mathrm{PAH}^{+}$cation. The key to this process is the methylene functional group $\left(\mathrm{CH}_{2}\right)$ in the superhydrogenated $(\mathrm{PAH}) \mathrm{H}^{+}$cation. In our calculations, we proved that metathesis of the naphthalene cation can occur with an energy barrier lower than that of the $-\mathrm{H}$ channel. This process leads to a cation (designated by the letter $\mathrm{H}$ in the Fig. 5) with local superhydrogenation which could be also involved in the $\mathrm{H}_{2}$ formation mechanism described above.
Acknowledgements. We thank Y. Ellinger, F. Pauzat and D. Talbi for their helpful discussion about the singlet and triplet states of the naphthyl cation.

\section{References}

Allamandola, L. J., Tielens, A. G .G. M., \& Barker, J. R. 1985, ApJ, 290, L25

Allamandola, L. J., Tielens, A. G. G. M., \& Barker, J. R. 1989, ApJS, 71,733

Bauschlicher, C. W. Jr 1998, ApJ, 509, L125

Bauschlicher, C. W. Jr 1999, ApJ, 517, L67

Bauschlicher, C. W. Jr, Hudgins, D. M., \& Allamandola, L. J. 1999, Theo. Chem. Acc., 103 (2), 154

Bauschlicher, C. W. Jr, \& Bakes, E. L. O. 2001, Chem. Phys., 274, 11

Becke, A. D. 1992, J. Chem. Phys., 97, 9173

Bernstein, M. P., Sandford, S. A., \& Allamandola, L. J. 1996, 472, L127

Boissel, P., de Parseval, P., Marty, P., \& Lefévre, G. 1997, J. Chem. Phys., 106, 4973

Frisch, M. J., et al. 1998, GAUSSIAN 98 (Rev. A.7, Pittsburgh: Gaussian, Inc.)

Geballe, T. R., Tielens, A. G. G. M., Allamandola, L. J., Moorhouse, A., \& Brand, P. W. J. L. 1989, ApJ, 341, 278

Gotkis, Y., Oleinikova, M., Naor, M., \& Lifshitz, C. 1993, J. Phys. Chem., 97, 12282

Granucci, G., Ellinger, Y., \& Boissel, P. 1995, Chem. Phys., 191, 165

Habart, E., Boulanger, F., Verstraete, L., Walmsley, C. M., \& Pineau des Forêts, G. 2004, A\&A, 414, 531

Hirama, M., Tokosumi, T., Ishida, T., \& Aihara, J.-I. 2004, Chem. Phys., 305, 307

Ho, Y. P., Dunbar, R. C., \& Lifshitz, C. 1995, J. Am. Chem. Soc., 117, 6504

Huber, K. P., \& Hertzberg, G. 1979, Molecular Spectra and Molecular Structure Constants of Diatomic Molecules (New York: Van Nostrand)

Joblin, C., Tielens, A. G. G. M., Allamandola, L. J., \& Geballe, T. R. 1996, ApJ, 458, 610

Joblin, C., Maillard, J. P., Vauglin, I., Pech, C., \& Boissel, P. 2000, in Molecular Hydrogen in Space, ed. F. Combes, \& G. Pineau des Forêts (Cambridge University Press), 107

Joblin, C., Pech, C., Armengaud, M., Frabel, P., \& Boissel, P. 2002, Proc. Infrared and Submillimeter Space Astronomy, held 1113 June, 2001, ed. M. Giard, J. P. Bernard, A. Klotz, \& I. Ristorcelli (EDP Sciences), EAS Publ. Ser., 4, 73

Joblin, C., Boissel, P., Armengaud, M., \& Frabel, P. 2005a, Int. J. Mass Spectrom., submitted

Joblin, C., Toublanc, D., Pech, C., et al. 2005b, in preparation

Jochims, H.W., Rasekh, H., Rühl, E., \& Baumgärtel, H. 1992, Chem. Phys., 168, 159

Langhoff, S. R. 1996, J. Phys. Chem., 100, 2819

Lee, A., Yang, W., \& Parr, R. G. 1988, Phys. Rev., B, 37, 785

Léger, A., \& Puget, J.-L. 1984, A\&A, 137, L5

Léger, A., d'Hendecourt, L., Boissel, P., \& Dśert, F. X. 1989a, A\&A, 213,351

Léger, A., d'Hendecourt, L., \& Defourneau, D. 1989b, A\&A, 216, 148

McMillen, D. F., \& Golden, D. M. 1982, Ann. Rev. Phys. Chem., 33, 493

Pauzat, F., Ellinger, Y., \& Talbi, D. 1993, in Molecules and Grains in Space, ed. I. Nenner, AIP Conf. Proc., 312, 635

Scott, A. P., \& Radom, L. 1996, J. Phys. Chem., 100, 16502

Sloan, G. C., Bregman, J. D., Geballe, T. R., Allamandola, L. J., \& Woodward, C. E. 1997, AJ, 474, 735

Wagner, D. R., Kim, H. S., \& Saykally, R. J. 2000, ApJ, 545, 854 